\title{
Antifungal and post-antifungal effects of chlorhexidine, fluconazole, chitosan and its combinations on Candida albicans
}

\author{
Silvia-Edith Calamari ${ }^{1}$, María-Alejandra Bojanich ${ }^{2}$, Silvina-Ruth Barembaum ${ }^{1}$, Nora Berdicevski ${ }^{2}$, Ana- \\ Isabel Azcurra ${ }^{1}$ \\ ${ }^{1}$ Regular Professor. Oral Biology Department, Faculty of Dentistry. National University of Córdoba-Argentina \\ ${ }^{2}$ Assistant Professor. Oral Biology Department, Faculty of Dentistry. National University of Córdoba-Argentina \\ ${ }^{3}$ Regular Professor. Oral Rehabilitation Department, Faculty of Dentistry. National University of Córdoba-Argentina
}

Correspondence:

Cathedra of Biological Chemistry

Oral Biology Department

Faculty of Dentistry

National University of Córdoba

Haya de la Torre S/N-Ciudad Universitaria

5006 Córdoba, Argentina

anaazcurra@yahoo.com

\begin{abstract}
Calamari SE, Bojanich MA, Barembaum SR, Berdicevski N, Azcurra AI. Antifungal and post-antifungal effects of chlorhexidine, fluconazole, chitosan and its combinations on Candida albicans. Med Oral Patol Oral Cir Bucal. 2011 Jan 1;16 (1):e23-8.

http://www.medicinaoral.com/medoralfree01/v16i1/medoralv16ilp23.pdf
\end{abstract}

Received: 13/10/2009

Accepted: 06/03/2010

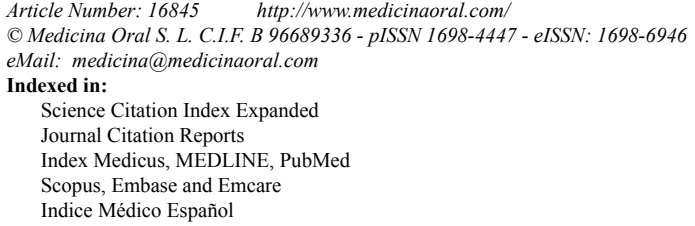

\begin{abstract}
Objective: The aim of this work was to assess the antifungal and post-antifungal effects of chlorhexidine, fluconazole, chitosan and its combinations on virulence factors of Candida albicans.

Study Design: Ten isolated strains of Candida albicans obtained from 10 patients with oral candidiasis and a collection strain of $C$. albicans were treated with antifungal agents in different concentrations or combinations of them. Virulence factors analyzed were the cell surface hydrophobicity, the germinative tube development, the phospholipase activity and the post-antifungal effect of that exposure.

Results: Virulence factors of the isolated strains obtained from patients together with the collection strain showed significant decreases with the different antifungal treatments, except for hydrophobicity and phospholipase activity. The development of germinative tube was the most sensitive factor to all the antifungal agents used. Untreated strains as well as the ones treated with antifungal agents showed a positive correlation among the virulence factors analyzed. No synergic effects arose from the combinations of the used drugs.

Conclusions: $C$. albicans isolated strains from patients showed high phospholipase activity and germinative tube production, which corroborates their capacity to infect the oral mucosa and the high prevalence of species. As a whole, our results imply that short exposures to sub-inhibitory concentrations of the antifungal agents under analysis, isolated or combined, can modulate the way virulence factors get manifested, thus decreasing their pathogenicity.
\end{abstract}

Key words: Candida albicans, chitosan, chlorhexidine, fluconazole, antifungal effect. 


\section{Introduction}

Candida genus is composed of more than 150 different species, but Candida albicans is the main responsible species for oral candidiasis. An increase in the percentage of colonized people has been observed according to their age and the presence of predisposing local or general factors $(1,2)$. The first stage of the infection includes adhesion to cellular surfaces. Adhesion of yeast has been studied using buccal epithelial cells, different cell lines, and plastic materials $(3,4)$, with the cell surface hydrophobicity of fungus (CSH) as the main force implied in this process (5). Besides, C. albicans is capable of secreting exoenzymes, such as phospholipase (PL), and of developing hyphae, which allows penetration in oral tissues and, in turn, the infection process $(6,7)$. Another form of measuring the yeast virulence is through the post antifungal effect (PAFE), which measures the growth recovery capacity after a limited exposure to antifungal agents (8).

In recent years, an increase of resistance to some antifungics agents has been observed and this has caused concern due to the eukaryotic character of Candida as well as of host cells, and due to a smaller number of available antifungal drugs compared to antibiotics (9). Some clinical research has used some chlorhexidinebased (CLX) oral rinses as an alternative to antifungal agents used regularly, obtaining good results (10); while other studies have shown an increase of pathogenicity in strains treated with fluconazole (FLZ) in a model of systemic infection in rats (11). Other authors have shown an important FLZ effect on the capacity of adhesion and production of exoenzymes (12). One of the new biomaterials being used in Dentistry is chitosan (HMWC), a cationic polymer obtained from chitin (13). The effect on inhibition of $C$. albicans fungal adhesion to epithelial cells and to hydroxyapatite pearls has been demonstrated in vitro, as well as its use as a vehicle or adjuvant in antifungal action (3, 14-16). Seyfarth et al. showed an existing positive correlation between chitosan molecular weight and its effectiveness as an antifungal agent in an in vitro study (17). In this sense, the combination of treatments could reduce the employed dose, thus preventing the development of antifungal resistance in the therapeutic treatment of this pathology.

In this work, antifungal and post- antifungal effects of CLX, FLZ, HMWC and its combinations were assessed on some virulence factors of C. albicans after a short exposure to these drugs in sub-inhibitory concentrations. Besides, the existing correlation between the mentioned factors was analyzed in the presence and in the absence of the antifungal agents under study.

\section{Materials and Methods}

Isolation and identification of 10 isolated wild C. albicans strains

The strains were isolated from the saliva of 10 patients with oral candidiasis, having complete or partial dentures, who were assisted at Cathedra of Prosthodontics III, Faculty of Dentistry, National University of Córdoba, Argentina; a collection strain was isolated as well (C.albicans serotype A NCPF 3153 - CA 3153 - kindly provided by Dr. Jose Ponton, University of the Basque Country, Spain). The patients agreed to sing a consent form prior to their participation in the study. Patients who were under treatment with antifungal drugs, xerostomic drugs, or under a prolonged treatment with anti-inflammatory drugs 10 days prior to exam were excluded. The protocol was approved by the Ethics Committee, Polo Hospitalario, Córdoba, Argentina. Samples were harvested in Sabouraud glucose agar (SGA) with $1 \%$ chloramphenicol (Britania, Argentina); identification of $C$. albicans species was carried out through germinative tube development test, sugar assimilation and fermentation (Candifast, International Microbe, France), and growth within a chromogenic medium (CHROMagar Candida, France) (18). The isolated yeasts were kept in sterile water at $4{ }^{\circ} \mathrm{C}$.

\section{Antifungal agents}

Chlorhexidine solutions were used, $12.5-25-50 \mu \mathrm{g} / \mathrm{ml}$ chlorhexidine (CLX, Microsules Bernabó, Argentina), as well as $50-100-150 \mu \mathrm{g} / \mathrm{ml}$ fluconazole (FLZ, Pfizer, Argentina), $0.25 \mathrm{~g} \%$ high-molecular-weight chitosan (HMWC $0,25 \%$ in acetic acid buffer $\mathrm{pH} 4,90 \%$ deacylated, $280 \mathrm{cps}$, PM $300 \mathrm{kDa}$, Unifarma Argentina) and their combinations (QAPM 0.25g\% / CLX $50 \mu \mathrm{g} / \mathrm{ml}$ and QAPM $0.25 \mathrm{~g} \%$ / FLZ $150 \mu \mathrm{g} / \mathrm{ml}$ ) in phosphate saline buffer (PBS) $\mathrm{pH}$ 7.4. These concentrations, which are sub-inhibitory for yeasts, were previously determined in the isolated strains in our laboratory, and proved to coincide with the ones employed in our previous work and in other authors' work $(16,19,20)$. Minimum inhibitory concentration (MIC) was determined using the agar dilution method, which defines MIC as the lowest antifungal drug concentration where a considerable decrease in the colony size is observed compared to control growth. This method has shown a good correlation with the reference method (21).

\section{Yeast suspension preparation for the trials}

Before the trials, isolated yeast strains were inoculated on SGA plates and incubated during $24 \mathrm{~h}$ at $37^{\circ} \mathrm{C}$. Subsequently, suspensions were prepared in sterile PBS (absorbance: 1.500 at $520 \mathrm{~nm}$ ). Then, $1 \mathrm{~mL}$ of suspension was added to $4 \mathrm{~mL}$ of PBS (control) or to $4 \mathrm{~mL}$ of PBS-antifungal agents in the aforementioned concentrations, resulting in a $1 \times 10^{6}-1 \times 10^{7}$ cells $/ \mathrm{mL}$ concentration. Tubes were incubated during $60 \mathrm{~min}$ at $37^{\circ} \mathrm{C}$ with gentle agitation. Cells were washed twice with sterile 
PBS (10 min at 3,000 rpm); later, they were resuspended in sterile PBS and adjusted to Abs520 nm $=0.800$. After this, the viability was verified on SGA plates. Besides, viability of all strains was verified after treatment with acetic acid buffer $\mathrm{pH} 4$; inhibition of growth was not observed.

Determination of cell surface hydrophobicity (CSH) Adhesion to hydrocarbons method was used to determine CSH. Yeast suspensions were mixed with chloroform (volume relationship 5:1); the mixture was agitated for 1 minute and put to rest during $10 \mathrm{~min}$ at $37^{\circ} \mathrm{C}$. CSH was defined as the difference between absorbances at $520 \mathrm{~nm}$ of the aqueous phase before and after the confronting C. albicans suspension and the organic phase, $\Delta$ Abs (5).

\section{Germinative tube development (GT)}

In order to induce GT development, $250 \mu \mathrm{l}$ yeast suspensions treated with antifungal drugs were mixed with $1 \mathrm{~mL}$ of human serum, and incubated at $37^{\circ} \mathrm{C}$ during 2.5 hours. Once that time elapsed, 300 cells in continuous fields (X 400) in Neubauer chamber were counted and GT cells were expressed as a percentage (6).

Determination of phospholipase activity $(P L)$

PL activity of $C$. albicans strains was determined by a modified version of Vidotto's technique (6). A 0.075 g\% soy lecithin suspension (Herbaccion Isa, Argentina) with $0.056 \mathrm{~g} \% \mathrm{CaCl} 2$ and $5.84 \mathrm{~g} \% \mathrm{NaCl}$ was used in each plate of SGA. $10 \mu \mathrm{l}$ of suspensions were inoculated in SGA supplemented with lecithin and incubated for $72-96$ hours at $37^{\circ} \mathrm{C}$. Each strain was tested in triplicate. The value of PL activity was determined as the quotient between the colony diameter plus halo and the colony diameter, $\mathrm{Pz}=(\mathrm{d}$ col $+\mathrm{d}$ halo $) / \mathrm{dcol}(7)$.

Determination of post antifungal effect (PAFE)

In order to study the inhibitory effect on the recovery of fungal growth of the analyzed compounds, namely PAFE, $800 \mu \mathrm{L}$ of $C$. albicans suspensions were incubated with $1.2 \mathrm{~mL}$ of RPMI 1640 (American Biorganics, USA) during $24 \mathrm{~h}$ at $37^{\circ} \mathrm{C}$ in a thermostatic bath with gentle agitation. Changes of absorbance at $520 \mathrm{~nm}$ were observed from the beginning of trial and after 24 $\mathrm{h}(\Delta \mathrm{Abs} 520 \mathrm{~nm})$. Thus, a greater value indicates a better recovery of the fungal growth and, therefore, a smaller PAFE (8). A period of 24 hours was selected as final time for absorbance record, after an experiment where the absorbance at $520 \mathrm{~nm}$ was recorded each hour, from time 0 to $32 \mathrm{~h}$.

All experiments were carried out in triplicate and data were statistically processed by ANOVA, Spearman's correlation coefficient and Student's t-test for independent samples $(\mathrm{p}<0.05)$.

\section{Results and Discussion}

Germinative tube (GT) development

In isolated $C$. albicans strains obtained from patients and treated with CLX, FLZ and HMWC, alone or combined with the aforementioned compounds, a significant decrease of GT was observed (Fig. 1-a). The collection strain, CA 3153, showed significant decreases in GT after treatment with antifungal drugs and its combinations, except for FLZ. The most significant decreases of GT were observed in the presence of CLX, which proved to be the most efficient antifungal agent regarding proportional reductions of GT with concentrations (data not shown), in agreement with results observed by Ellepola et al. (22).

Cell surface hydrophobicity (CSH)

A significant $\mathrm{CSH}$ increase in isolated strains from patients was observed (Fig. 1-b) and also in CA 3153 after treatment with HMWC, HMWC/CLX and HMWC/ FLZ. No significant differences were observed after treating strains only with CLX or FLZ. These results do not agree with those observed by Anil et al. (20) and ours on previous works (3), although employing the same concentrations $(50 \mu \mathrm{g} / \mathrm{ml})$. This could be explained by the differences in the experimental conditions, since in the present study yeast suspensions are within a solution in contact with antifungics in short times, which implies contact on cell surface and would represent a similar situation as the one occurring during the use of oral rinses. Goldberg et al. (23) found results similar to ours in the presence of HMWC, assigning the CSH increase to the polycationic character of chitosan, and this would cause a decrease of superficial negative charge of yeasts and the subsequent increase of cellular hydrophobicity $(17,23)$. Lack of effect on CSH when strains were treated with antifungics in the absence of HMWC indicates that the polycationic polymer would be responsible for the observed effect, without synergism when combined with CLX and FLZ.

Determination of post antifungal effect (PAFE)

Isolated strains from patients and CA 3153 showed a significant decrease in the growth recovery when they were treated with CLX (Figure 1-c). FLZ produced a significant decrease of CA 3153 growth recovery. In the presence of HMWC, alone or combined with FLZ, an increasing tendency in the growth recovery was observed. These results show that the significant decrease observed in the presence of CLX or FLZ is counteracted in the presence of HMWC, the effect of which prevails when antifungal drugs are combined with it. Previous results have shown the antifungal effect of dissolved chitosan within the solid medium of yeast growth (3); nevertheless, the experimental conditions of this work have been different, as it has been discussed in a previous section; this is likely to be this way due to the short period contact, similarly to what happens in the case of an oral rinse. 
Determination of phospholipase activity ( $P L)$

All isolated $C$. albicans strains obtained from patients and CA 3153 exhibited PL activity. When they were treated with the analyzed antifungal agents, CA 3153 showed a significant decrease of PL activity in the presence of CLX, which proved directly proportional to concentrations, in accordance with Kadir et al's findings (7). In isolated strains from patients no differences were observed in PL activity with the different treatments (Fig. 1-d). This difference in the behavior between isolated strains from patients and CA 3153 may arise from the fact that these, due to their wild condition, could be adapted more easily through other mechanisms to overcome environmental difficulties or host immunity mechanisms, resulting in more resistant strains when treated with antifungal drugs.

Correlation among the analyzed virulence factors

A strong positive correlation among the analyzed virulence factors was observed in isolated untreated strains $\left(r^{2}>0.73\right.$ and $\left.p<0.03\right)$. These results corroborate the cooperative effect of these factors, which is a breeding ground for the development of yeast virulence and, in

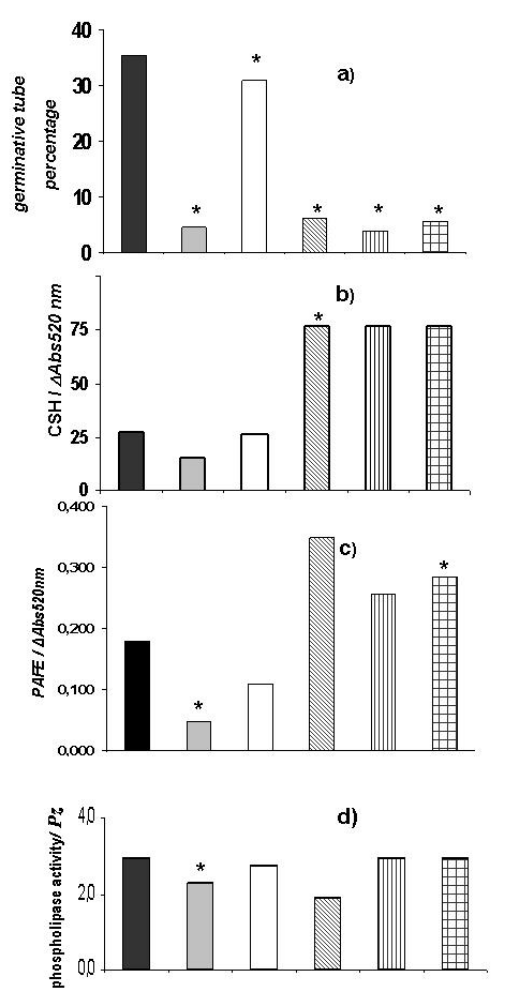

Fig. 1. Effect of chlorhexidine (CLX), fluconazole (FLZ), high molecular weight chitosan (HMWC) and its combinations on virulence factors of $\mathrm{C}$. albicans isolated from patients. a) germinative tube development percentage, GT b) cell surface hydrophobicity, $\mathrm{CSH}$, as $\triangle \mathrm{Abs} 520 \mathrm{~nm}$ c) post-antifungal effect, PAFE, as $\triangle \mathrm{Abs} 520 \mathrm{~nm}$ d) phospholipase activity, $\mathrm{Pz}$.

* indicates significant differences respect to control; $\mathrm{p}<0.05$.

CONTROL $\square \mathrm{CLX} \square \mathrm{FLZ}$ HMWC 四HMWC+CLX 田 HMWC+FLZ turn, the illness, in accordance with Vidotto et al. (6). Other authors (24) have shown the coordinated action of virulence factors, such as the contribution to fungal nutrition and the micro-environmental modification produced by fatty acid liberation from fungal lipase activity. Such liberation benefits exoenzymes proteinases activity, producing a $\mathrm{pH}$ decrease to values between 5.5 and 2.8. Additionally, fatty acid liberation improves $C$. albicans adhesion to host surfaces. Besides, Masuoka et al. correlated the composition of $C$. albicans cell wall (long of chain of the acid-labile portion) with change in the fungal hydrophobicity (25).

When treating yeasts with the analyzed antifungics, CLX produced positive correlations among all the factors $(\mathrm{p}<0.002)$; and FLZ produced the same effect on GT, PAFE and PL activity $(\mathrm{p}<0.02)$. HMWC produced strong positive correlations $(\mathrm{p}<0.02)$, except between PAFE and PL activity. When combining HMWC with CLX, a strong positive correlation was observed between the factors $\left(\mathrm{r}^{2}>0.7-\mathrm{p}<0.002\right)$, except for GT and PAFE, while HMWC combined with FLZ did not produce significant associations. The combined use of antifungal drugs has been evaluated by many authors. Senel et al. (in vitro studies) (26) and Giunchedi et al. (in vivo studies) (27) obtained good results, showing that chitosan produced an adjuvant effect on liberation and effectiveness of drugs to which the former was associated in those studies. In accordance with our results, Ellepola et al. showed a strong correlation between virulence factors after treating C. albicans strains with CLX (22). Nevertheless, CLX is not habitually used by clinicians in our country for oral candidiasis treatment, considering its effects on the sense of taste and the staining of oral tissues and acrylic dentures (28). In this work, after combining HMWC with FLZ, a drastic effect on the capacity for GT development was observed. Considering the fact that GT development implies not only morphological changes but also a greater capacity for infection and penetration of oral soft tissues, this work contributes evidences to the results obtained by other authors in this area.

The use of virulence factors as gravity predictors of fungal infections or as in vitro models for studying C. albicans behavior should be carefully analyzed. Though our results show that GT development is very sensitive to the antifungal treatment, it should not be considered as an only parameter or predictor of fungal virulence. The strong correlation observed in the fungus virulence factors involved in infections shows that an individual evaluation of them would be a simplification of the analysis, considering that not all the factors are expressed in infections or necessarily in a particular stage of infection (29).

Ellepola et al. have shown that a combined treatment with antifungics would act as adjuvants in denturerelated oral candidiasis and would allow diminishing 
medicine doses, thus preventing the development of antifungal resistance (22). These authors conclude that clinical manifestations in oral candidiasis and recent advances in antifungal generation give a new clinical meaning to the post-antifungal effect concept, considering it as an in vitro model for evaluation of therapeutic treatment in the future.

Although intra-oral concentrations of antifungals can fluctuate considerably in oral cavity, our results indicate that sub-inhibitory concentrations of antifungics used diminish the infection capacity of $C$. albicans, represented by virulence factors, in accordance with other authors (7). In clinical terms, our results would indicate that short exposures to sub-inhibitory concentrations with the analyzed drugs would be able to modulate the expression of the virulence factors studied and therefore, to facilitate the elimination of oral cavity microorganisms, and to decrease Candida's patogenicity, in spite of the brief exposure period in the oral environment.

\section{Conclusions}

The present work was based on the effect that short exposures of antifungals to sub-inhibitory concentrations has on virulence factors of $C$. albicans, the most important etiologic agent of oral candidiasis. Our results suggest that adhesion, represented by the evaluation of $\mathrm{CSH}$, exoenzymes production, dimorphism and growth recovery after treatment with antifungals in short periods, can be factors that add to the predisposing conditions of host and, in turn, determine the development of candidiasis or the status of patients as healthy carriers. On the other hand, this study confirms that antifungics used would be able to modulate the development of candidiasis.

\section{References}

References with links to Crossref - DOI

1. Lopez-De-Blanc SA, Salati-De-Mugnolo N, Femopase FL, Benitez MB, Morelatto RA, Astrada-De-Verde L, et al. Antifungal topical therapy in oral chronic candidosis. A comparative study. Med Oral. 2002;7:260-70.

2. Kliemann DA, Pasqualotto AC, Falavigna M, Giaretta T, Severo LC. Candida esophagitis: species distribution and risk factors for infection. Rev Inst Med Trop Sao Paulo. 2008;50:261-3.

3. Azcurra AI, Barembaum SR, Bojanich MA, Calamari SE, Aguilar $\mathrm{J}$, Battellino LJ, et al. Effect of the high molecular weight chitosan and sodium alginate on Candida albicans hydrophobicity and adhesion to cells. Med Oral Patol Oral Cir Bucal. 2006;11:E120-5.

4. Baena-Monroy T, Moreno-Maldonado V, Franco-Martínez F, Aldape-Barrios B, Quindós G, Sánchez-Vargas LO. Candida albicans, Staphylococcus aureus and Streptococcus mutans colonization in patients wearing dental prosthesis. Med Oral Patol Oral Cir Bucal. 2005;10 Suppl 1:E27-39.

5. Singleton DR, Masuoka J, Hazen KC. Surface hydrophobicity changes of two Candida albicans serotype B mnn4delta mutants. Eukaryot Cell. 2005;4:639-48.

6. Vidotto V, Yumi Koga-Ito C, Milano R, Fianchino B, Pontón J. Correlation between germ tube production, phospholipase activity and serotype distribution in Candida albicans. Rev Iberoam Micol. 1999;16:208-10.

7. Kadir T, Gümrü B, Uygun-Can B. Phospholipase activity of Can- dida albicans isolates from patients with denture stomatitis: the influence of chlorhexidine gluconate on phospholipase production. Arch Oral Biol. 2007;52:691-6.

8. Egusa H, Ellepola AN, Nikawa H, Hamada T, Samaranayake LP. Sub-therapeutic exposure to polyene antimycotics elicits a post-antifungal effect (PAFE) and depresses the cell surface hydrophobicity of oral Candida albicans isolates. J Oral Pathol Med. 2000;29:20613.

9. Denning DW, Hope WW. Therapy for fungal diseases: opportunities and priorities. Trends Microbiol. 2010;18:195-204.

10. Barasch A, Safford MM, Dapkute-Marcus I, Fine DH. Efficacy of chlorhexidine gluconate rinse for treatment and prevention of oral candidiasis in HIV-infected children: a pilot study. Oral Surg Oral Med Oral Pathol Oral Radiol Endod. 2004;97:204-7.

11. Navarathna DH, Hornby JM, Hoerrmann N, Parkhurst AM, Duhamel GE, Nickerson KW. Enhanced pathogenicity of Candida albicans pre-treated with subinhibitory concentrations of fluconazole in a mouse model of disseminated candidiasis. J Antimicrob Chemother. 2005;56:1156-9.

12. Lyon JP, De Resende MA. Correlation between adhesion, enzyme production, and susceptibility to fluconazole in Candida albicans obtained from denture wearers. Oral Surg Oral Med Oral Pathol Oral Radiol Endod. 2006;102:632-8.

13. Koide SS. Chitin-chitosan: properties, benefits and risks. Nutrition Research. 1998;18:1091-101.

14. Barembaum S, Virga C, Bojanich A, Cornejo L, Calamari S, Pontón J, et al. Effect of Chitosan and Sodium Alginate on the adherence of autochthonous C. Albicans to oral epithelial cells (in vitro). Med Oral. 2003;8:188-96.

15. Rossi S, Sandri G, Ferrari F, Bonferoni MC, Caramella C. Buccal delivery of acyclovir from films based on chitosan and polyacrylic acid. Pharm Dev Technol. 2003;8:199-208.

16. Calamari S, Bojanich A, Barembaum S, Azcurra A, Virga C, Dorronsoro S. [High molecular weight chitosan and sodium alginate effect on secretory acid proteinase of Candida albicans]. Rev Iberoam Micol. 2004;21:206-8.

17. Seyfarth F, Schliemann S, Elsner P, Hipler UC. Antifungal effect of high- and low-molecular-weight chitosan hydrochloride, carboxymethyl chitosan, chitosan oligosaccharide and N-acetyl-Dglucosamine against Candida albicans, Candida krusei and Candida glabrata. Int J Pharm. 2008;353:139-48.

18. García-Martos P, García-Agudo R, Hernández-Molina JM, Marín P, Tallero E, Mira J. [Identification of yeasts of clinical interest on CHROMagar Candida culture medium.]. Rev Iberoam Micol. 1998;15:131-5.

19. Carrillo-Muñoz AJ, Quindós G, Ruesga M, Alonso R, Del Valle $\mathrm{O}$, Hernández-Molina JM, et al. Antifungal activity of posaconazole compared with fluconazole and amphotericin B against yeasts from oropharyngeal candidiasis and other infections. J Antimicrob Chemother. 2005;55:317-9.

20. Anil S, Ellepola AN, Samaranayake LP. The impact of chlorhexidine gluconate on the relative cell surface hydrophobicity of oral Candida albicans. Oral Dis. 2001;7:119-22.

21. Yoshida T, Jono K, Okonogi K. Modified agar dilution susceptibility testing method for determining in vitro activities of antifungal agents, including azole compounds. Antimicrob Agents Chemother. 1997:41:1349-51.

22. Ellepola AN, Samaranayake LP. The effect of brief exposure to sub-therapeutic concentrations of chlorhexidine gluconate on the germ tube formation of oral Candida albicans and its relationship to post-antifungal effect. Oral Dis. 2000;6:166-71.

23. Goldberg S, Doyle RJ, Rosenberg M. Mechanism of enhancement of microbial cell hydrophobicity by cationic polymers. J Bacteriol. 1990;172:5650-4.

24. Hube B, Stehr F, Bossenz M, Mazur A, Kretschmar M, Schäfer W. Secreted lipases of Candida albicans: cloning, characterisation and expression analysis of a new gene family with at least ten members. Arch Microbiol. 2000;174:362-74.

25. Masuoka J, Hazen KC. Cell wall mannan and cell surface hy- 
drophobicity in Candida albicans serotype A and B strains. Infect Immun. 2004;72:6230-6.

26. Senel S, Ikinci G, Kaş S, Yousefi-Rad A, Sargon MF, Hincal AA. Chitosan films and hydrogels of chlorhexidine gluconate for oral mucosal delivery. Int J Pharm. 2000;193:197-203.

27. Giunchedi P, Juliano C, Gavini E, Cossu M, Sorrenti M. Formulation and in vivo evaluation of chlorhexidine buccal tablets prepared using drug-loaded chitosan microspheres. Eur J Pharm Biopharm. 2002;53:233-9.

28. Ellepola AN, Samaranayake LP. Adjunctive use of chlorhexidine in oral candidoses: a review. Oral Dis. 2001;7:11-7.

29. Pinto E, Ribeiro IC, Ferreira NJ, Fortes CE, Fonseca PA, Figueiral $\mathrm{MH}$. Correlation between enzyme production, germ tube formation and susceptibility to fluconazole in Candida species isolated from patients with denture-related stomatitis and control individuals. J Oral Pathol Med. 2008;37:587-92. 